\title{
SHEDDING LIGHT ON GAME ENGINES AND VIRTUAL REALITY FOR DESIGN IDEATION
}

\author{
Ekströmer, Philip; Wever, Renee; Andersson, Patrik; Jönsson, Johan \\ Linköping University
}

\begin{abstract}
While pen-and-paper sketches is generally considered the best tool for design ideation, there are certain areas of design where the ideas being generated do not easily lend themselves to sketching. This study reports on two cases that explores the use of game engines in combination with Virtual Reality (VR) to visualize lighting in the automotive industry. In the first case, the exterior lights of a car were visualized using Unreal Engine 4 and evaluated using research through design and expert interviews. In the second case, Unreal Engine VR Editor was used to explore ideation and concept development of interior lighting in long haulage trucks. The insights from the cases suggest that game engines and VR can be used to quickly develop and display ideas, concepts and scenarios in the early phases of the lighting design process. These strengths suggest that game engines and VR also have the potential to support design ideation for other types of design.
\end{abstract}

Keywords: Unreal Engine, Conceptual design, Product modelling / models, Creativity

\section{Contact:}

Ekströmer, Philip

Linköping University

Department of Management and Engineering

Sweden

philip.ekstromer@liu.se

Cite this article: Ekströmer, P., Wever, R., Andersson, P., Jönsson, J. (2019) 'Shedding Light on Game Engines and Virtual Reality for Design Ideation', in Proceedings of the 22nd International Conference on Engineering Design (ICED19), Delft, The Netherlands, 5-8 August 2019. DOI:10.1017/dsi.2019.206 


\section{INTRODUCTION}

The use of pen-and-paper sketches for design ideation is commonplace in most design practices. However, there are areas of design where the end products are abstract and do not easily lend themselves to sketching, which complicates the ideation process. One such area is automotive lighting design. Automotive headlamps, "the eyes of the car", are one of the most valuable brand assets on the front of the vehicle (Wördenweber et al., 2007). But while the headlamp is straightforward to sketch and is done so by car designers, sketching how the headlight will look and feel is very difficult. For this, Computer Aided Design (CAD) tools are much more effective, as many 3D graphics tools can produce accurate renderings of light. However, the iteration time is long as light is computationally intensive to render. Therefore, using game engines, software for game development using real-time rendering, in early stages of the lighting design process could have the potential to reduce iteration time and give the designers the opportunity to try more concepts. In this paper, the use of game engines and Virtual Reality (VR) tools for automotive lighting design ideation is explored by using parts of the master theses by Andersson (2017) and Jönsson (2017) as cases. In the first case, an exterior lighting visualization was developed to assess whether visualizations from a game engine, in this case Unreal Engine 4 (UE4), has any use for exterior lighting design. The second case explored the use of Unreal Engine VR Editor (UE VR) for ideation and concept development of interior lighting in long haulage trucks.

\section{BACKGROUND}

This section presents a short introduction to automotive lighting followed by literature related to the use of game engines and VR for design ideation.

\subsection{Automotive lighting}

Exterior lights are primarily designed to make the vehicle visible in traffic and illuminating the road ahead. However, they are also important design features when it comes to the design expression of the vehicle and the brand image (Wördenweber et al., 2007). It is therefore a common design strategy in the automotive industry to make small variations, face-lifts, of parts such as the headlamps, which create a differentiation between generations of models and feeling of newness for consumers (Andersson, 2016). The main purpose of the interior lighting is to facilitate orientation in the vehicle. Interior lighting also plays an important role in improving the feeling of safety and security (Wördenweber et al., 2007).

\subsection{Game engines}

Game engines are increasingly explored for different design applications. While originally created for game development, game engines support advanced visualizations and interactions which enable them to be used in other areas, such as construction and architectural design (Kosmadoudi et al., 2013). Game engines have been used in architecture and construction since the late 1990s to visualize environments, buildings and interiors (Shiratuddin and Thabet, 2002). Two early examples are Shiratuddin and Tabet (2002) who used Unreal Tournament engine to create an affordable and realistic three dimensional virtual walkthrough of an office space and Indraprastha and Shinozaki (2009) who studied the advantages and limitations of game engines for visual representations in urban architectural design. Later examples include Magica (2014), who developed an application for the design of cruise ships interiors and services using the game engine Unity 3D. The application was designed for rapid prototyping and testing in the early stages of design, giving designers the freedom to switch between prototyping and testing in immersive VR. Also in the maritime sector, Hjelseth, Morrison and Nordby (2015) studied how game engines can be used to facilitate the design process and how they may be used as design tools in a user scenario development for complex systems. They created three scenarios regarding safety in the maritime sector in the game engine CryEngine and evaluated them together with several experts in different fields, using co-design and participatory design methods. Hjelseth, Morrison and Nordby (2015) concluded that game engines enable designers to create scenarios with great complexity which can be used to trigger reflection-in-action (Schön, 1991) for both designers and users.

\subsection{Virtual reality}

Virtual reality and game engines have a close connection, as the latter is often used to create the immersive experience for use in VR. In a pilot study on Gestalt principles in product aesthetics, 
Valencia-Romero and Lugo (2017) used a game engine in combination with VR to explore the evaluation of aesthetics in an interactive and realistic setting. While the tools used were not the main focus of the study, they found that the advantages of virtual studies included that no real prototypes have to be made, thus reducing the cost of the evaluation, and the ability to automate the both presentation of choice sets and recording of responses.

In the automotive industry, VR has recently been employed for design concept validation. By combining the VR experience with a basic physical model of the car interior, both visibility and reachability studies can be improved (Holmes, 2017). McLaren Automotive have partnered up with Epic Games, developers of Unreal Engine, to implement VR in their development process. According to Mark Roberts, design operations manager at McLaren Automotive, using VR when comparing different interior designs is superior to physical mock-ups, as VR offers the same sense of space as the physical mock-up but allows much faster switching between concepts (Holmes, 2017).

\section{METHOD}

Both cases highlighted in this paper feature exploratory research with a research through design approach. Research through design is one of several ways to denote designerly ways of doing research (Stappers and Giaccardi, 2017). Using this methodology, the researcher generates new knowledge by creating specific solutions, allowing the designers to make discoveries through their own design process (Stappers and Giaccardi, 2017). Thus, during development of the exterior lighting visualization and interior lighting concepts respectively, the case authors reflected on the chosen tool and the problem situation and provided solutions and suggestions to the development process of exterior and interior automotive lighting. The knowledge gained from the design process was supplemented with user studies in both cases. Case 1 used four experts to evaluate the final visualization and Case 2 used six employees at RCDE to evaluate VR for ideation on interior lighting.

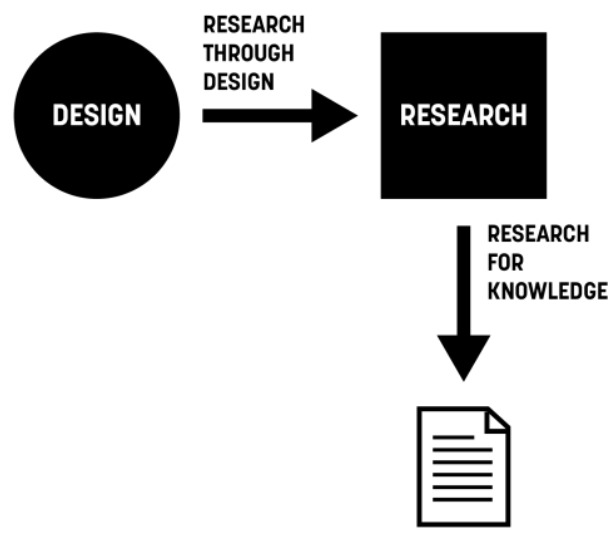

Figure 1. Research through design model adapted from Stappers and Giaccardi (2017).

\subsection{CASE 1: Developing an interactive lighting visualization}

The aim of this case was to explore the use of game engines to visualize early exterior lighting concepts. For this purpose, a visualization of a car was created. The main goals of the visualization were to create realistic looking lights, including low beam and high beam headlights, daytime running lights, turning signal lights, tail lights and brake lights, that could all be switched on and off, as well as a rich environment displaying the car in a realistic setting.

To create the visualization, a 3D model of a Honda CR-Z was used. The model was prepared for 3Ds Max and VRAY and free for commercial use. Materials were applied to make it look realistic and visual scripting was used to make behaviours and interactive elements. Most effort was put into the settings of the daytime running lights, brake lights and xenon high beam headlights to make their appearance realistic. The light effect was achieved by applying an emissive material, a type of material that emits light across the surface area, to all exterior lights. While there are pre-made lights in UE4, emissive materials were deemed sufficiently realistic for this visualization. However, after importing the model into UE4, it was discovered that it lacked a brake light above the trunk door. Thus, several configurations of this light were designed and tested during development. This was done by modifying 
a rectangle that had an emissive material applied to it. Three different configurations from the development process are shown in Figure 2 below.

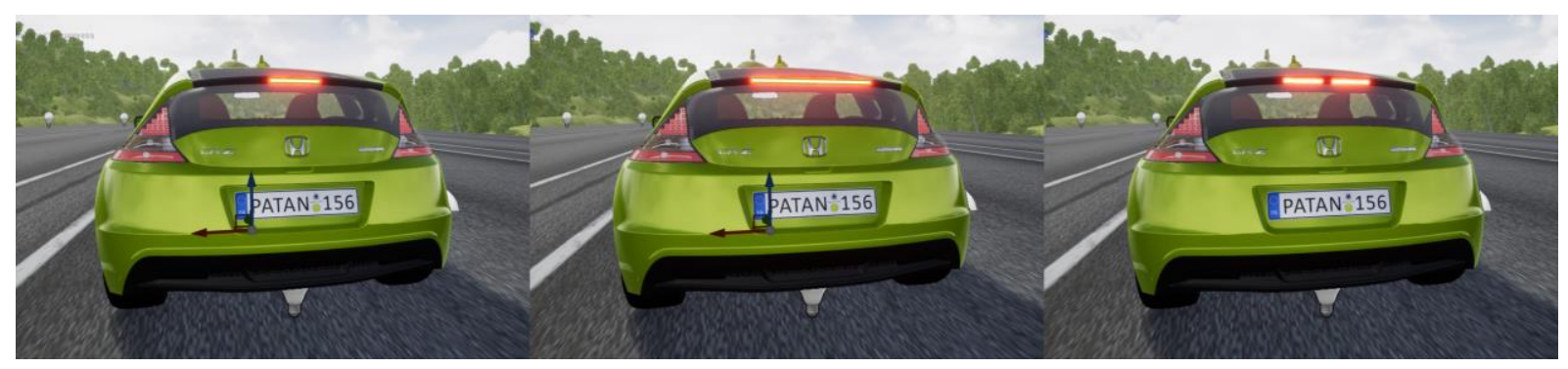

Figure 2 - Different iterations of the design of a break light above the trunk door

To further enhance the experience, two interactive scenarios were created. In the first scenario, a spotlight below the trunk door lit up when the player approached the vehicle from behind. This was achieved by using a box trigger, an object that activates a behaviour when it is interacted with by the player. In the second scenario, a spotlight switched on below the driver door as the door was opened. This is shown in Figure 3 below.

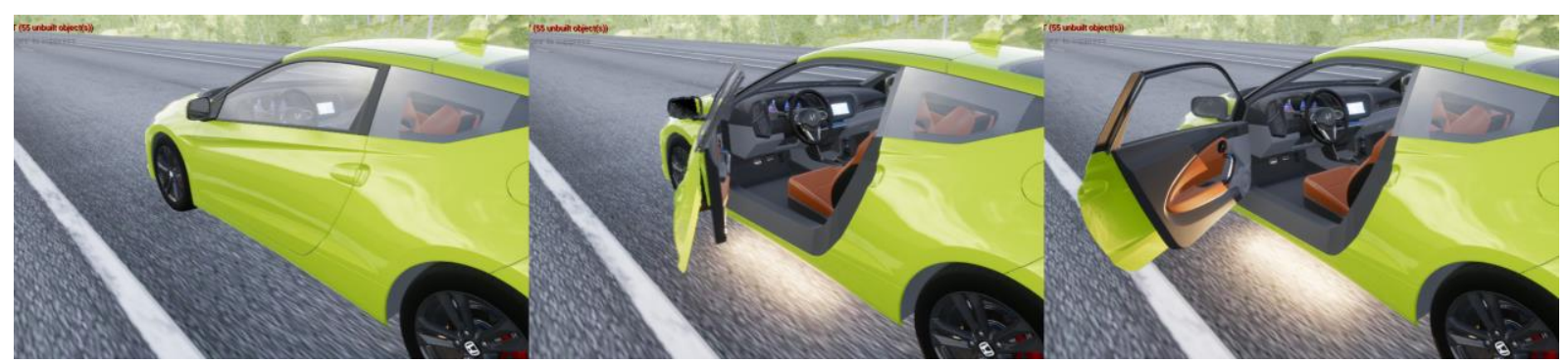

Figure 3-Image sequence showing the spotlight under the driver door. The spotlight is turned on when the player moves close to the door.

\subsection{Expert evaluation}

After the visualization was completed it was evaluated by four experts: a professor in advanced automotive design, a senior design engineer in exterior lighting systems, a manager of design visualization and a senior visualization designer in advanced automotive design. The experts were shown the UE4 interface, possible actions and other functionalities, as well as the following scenarios:

- A rear brake light that could be scaled and moved to create different designs

- How to quickly create a light below the passenger door

- A light below the trunk door being activated by the player moving close to the door

- Environmental light turned to night mode

- The car lights being turned on and off

All experts agreed that this had most potential for evaluating positioning, size or appearance of objects in early lighting design. The expert designer found the level of realism enough for the purpose of making design decisions in the early phases of developing exterior lighting. When shown the interactive light below the trunk door, he thought that it could allow for entirely new ways of evaluating design, even beyond lighting design. Further, he estimated that it would not be too much work for designers to learn the software as it is quite similar to other 3D-modeling software used in industry.

Both engineering and visualization deemed the fidelity of the visualizations too low for their use. They required more realistic visuals and, most importantly, a correlation study to determine how well the lights seen on screen represents reality. All the experts saw potential in using visualizations like this for evaluating brand recognition but maintained that it would require a much more advanced environment scene with other vehicles and certainty that what is seen on the screen corresponds to reality as mentioned earlier. Furthermore, all experts saw potential in using game engines for other purposes than evaluating exterior lighting design. 


\subsection{CASE 2: Exploring virtual reality for ideation on interior lighting}

The aim of the second case was to evaluate the use of VR tools for interior lighting design. This was done by using and evaluating UE VR, an VR interface for UE4 which allows the user to manipulate, create and experience the world in one-to-one scale, for ideation, concept creation and user evaluation. Only ideation and concept creation are presented in this paper. Ideation was evaluated through a user test, where six employees at Scania Physical Vehicle Ergonomics (RCDE) used UE VR to generate ideas for a relaxing lighting environment in the resting area of long haulage trucks. Concept creation was evaluated by the case author using UE VR to create interior lighting concepts as a part of the research through design methodology.

\subsection{Ideation}

To prepare for the ideation test, a virtual truck environment was created in UE4. This was done by modifying a default map, removing unnecessary objects, adding a model of a long haulage truck cabin, decorating with rocks and bushes and changing daytime settings to night, as illustrated in Figure 4.

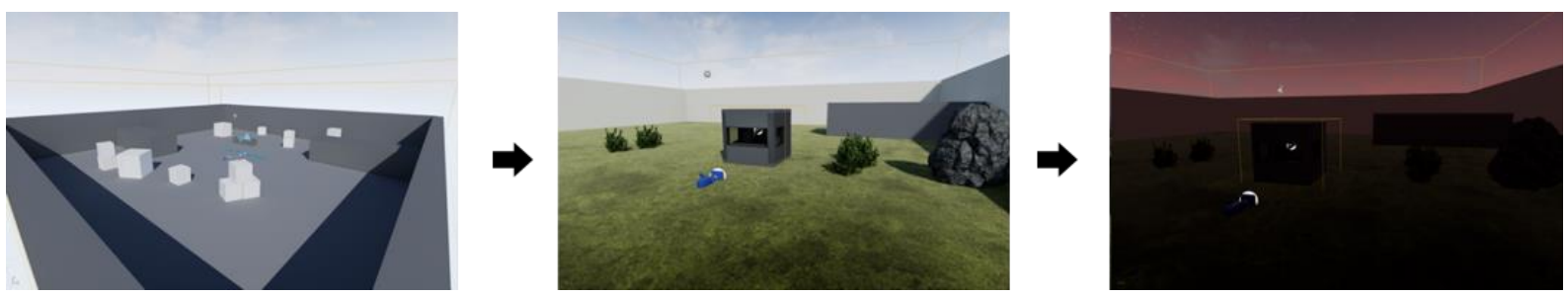

Figure 4. Creating the environment for ideation on interior lighting of truck cabins. The leftmost image shows the default map in UE4. The middle image shows the same map after adding environment materials and the truck cabin and decorating with rocks and bushes. The rightmost image shows the scene after daytime settings have been changed to night.

During the one-hour test session, the participants used an HTC VIVE headset to perform and evaluate four tasks using UE VR, with an extra fifth task if time allowed for it. The tasks were 1) Position light sources, 2) Increase and reduce number of light sources, 3) Change settings of light source, 4) Change IES-profile and document result and 5) Create a relaxing lighting environment for the resting area. Each step, except the last, began with a tutorial of the necessary interactions. After each task, one or two questions were asked about the experience. When all steps were done, or the time was up, eight questions regarding the entire experience were asked.

Overall, the participants found the experience of using UE VR positive. This was both reflected in answers and in observations by the case author. The participants expressed that this kind of workflow would allow for cheaper and easier exploration, especially when working with big vehicles like buses that requires more light sources. The ability to change light settings, allowing for several different characteristics from one light source, was highlighted as the main benefit of the experience, allowing more variations to be explored in one session. The participants also found that the limitless amounts of light sources gave them the feeling of having more possibilities. The ability to take screenshots directly within the virtual environment was regarded as very beneficial, as it is very hard to photograph light in reality. This was considered beneficial for conceptual design, as it makes it easy to show and share design suggestions.

The main concern voiced by the participants was the fidelity of the visualizations displayed in VR. Further, they thought that the virtual setting introduces a risk of creating lighting situations that might not be feasible. However, they argued that even if the concept is not possible to realize, it is easy to communicate and could be used as a vision.

When observing the interaction, it was clear that the participants learned how to interact in VR very quickly. Three of the six participants had time to do the fifth step, where they freely designed the lighting for the resting area of the truck. Also, it was interesting to note that the participants had no problem coming up with design concepts, despite the short amount of time and using UE VR for the first time.

\subsection{Concept generation}

The aim of the concept generation was both to further explore concept generation with UE VR and to prepare concepts for the user evaluation. To facilitates the creation and testing of different concepts, a 
custom environment was created. The test environment from the ideation test was reused and altered to fit the purpose, including three truck models instead of one and a camera switching system, allowing for quick switching between the three truck models.
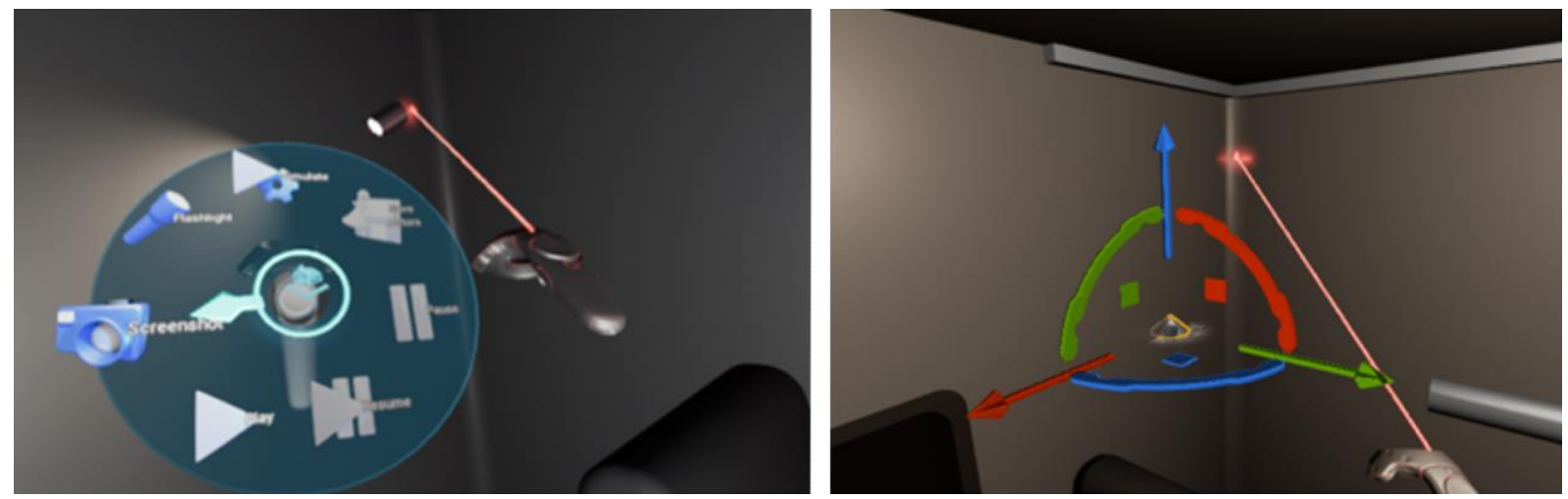

Figure 5. Using UE VR for concept generation. The left image shows the interface in the VR editor. The right image shows how geometry can be moved around in the environment.

The scenario which formed the basis from which to generate concepts was "Lighting to stimulate relaxation of the driver". When creating the concepts, basic geometry was modified to estimate shape, size and positioning of the lighting fixtures. Further, the reachability of one concept, where a light strip was placed above the driver bed, was evaluated by lying down in the same height as the bed with the VR headset on and trying to reach the fixture with the hand controller.

\section{DISCUSSION}

One of the most promising results was ease with which simple geometry could be placed and manipulated in UE4, both with and without VR. As the results are shown instantly, it enables for reflective conversation (Schön, 1991). In Case 1, this was evident when several variants of a new break light for the car could be quickly created by using a rectangle with emissive material and experimenting with the length and configurations. This further corroborates Hjelseth, Morrison and Nordby's (2015) suggestion that ideation in a game engine can trigger reflective conversation.

The main concern voiced in both cases was the fidelity of the visualizations. However, both the professor in advanced automotive design in Case 1 and the participants from RCDE in Case 2 considered what they were shown enough for design ideation. Moreover, the visualization and engineering experts in Case 1 argued for a correlation study to determine how well the virtual lights represents reality. While correlation studies could be undertaken, the result would not be relevant for very long since the capabilities of game engines are constantly updated, especially regarding lighting. Also, the technology of VR headsets is advancing rapidly with each new version having better resolution, field of view and optical accuracy, all of which are very relevant factors for the correlation result.

While the process of using a game engine and VR for ideation was quite fast and flexible in both cases, the process of preparing 3D-models for good results can be very difficult and time consuming. However, the quality requirements could be reduced for ideation which would shorten the time to get it ready. Moreover, Epic Games has recently released the workflow toolkit Datasmith, which promises reduced iteration time through efficient transfer of CAD data into UE4, for beta testing.

One of the downsides of game engines is that they require significant programming skill. This was evident in Case 1 when adding realistic weather effects proved to be too difficult. Hjelseth, Morrison and Nordby (2015) reflected that a team containing both a designer and a programmer would be preferable, but that this might make the workflow too complicated for design work. Interactivity is also quite time consuming to create and might hinder the fast and explorative workflow that is needed in early design. But it was considered very beneficial in Case 1 and might add realism to later stages when the product is being evaluated. Thus, interactivity might not be effective for the early exploration, but has shown potential in evaluation of lighting design and possibly also other design applications.

\section{CONCLUSIONS}

This paper has reported on two cases where UE4 and UE VR was used to develop and evaluate lighting design in early stages of the design process. The two cases suggest that game engines, both 
with and without VR, can be used to support ideation of exterior and interior lighting. Unreal Engine 4 can be used as a quick and easy way of displaying ideas, concepts or scenarios and UE VR allows for quick testing of different layouts, principles and ideas, that can easily be communicated and understood. The strengths of the tools highlighted in this study, as well as the feedback from the respondents in the cases, suggest that game engines and VR also have a use in design ideation outside the area of automotive lighting.

\section{FURTHER STUDIES}

While the design process is very much a team activity, with a heavy focus on collaboration, the VR headsets of today are promoting a solo experience. Thus, this calls for more studies on how collaborative VR, where all team members are able to be present in the same VR environment, will benefit early design ideation. This would introduce a group perspective, which was not touched upon in this study.

When developing the visualization in Case 1, it was discovered that it is possible to create camera settings in UE4 that simulates the degeneration of the eyes perception with age or certain eye defects. This was only quickly tested in the development process but could potentially be used in combination with VR to help a design team understand how the lighting is experienced under these conditions. It could also be used in other settings to get a feel for how products or interfaces are being perceived by users with sight defects. This would however, as mentioned by the experts in Case 1, require more studies to ensure that that what is seen on the screen or in VR represent or can be reliably translated to physical aspects of the real world.

\section{REFERENCES}

Andersson, P. (2017), Exterior Lighting Design in the Automotive Industry, Linköping University.

Andersson, T. (2016), Aesthetic Flexibility: Modularity of Visual Form in Product Portfolios and Branded Products, Linköping University.

Hjelseth, S., Morrison, A. and Nordby, K. (2015), "Design and Computer Simulated User Scenarios: Exploring Real-Time 3D Game Engines and Simulation in the Maritime Sector", International Journal of Design, Vol. 9 No. 3, pp. 63-75.

Holmes, S. (2017), "Everything is for a reason", Develop3D Magazine, available at: https://www.develop3d.com/features/everything-is-for-a-reason-McLaren-virtual-reality-VR-automotivedesign-CAD (accessed 10 December 2018).

Indraprastha, A. and Shinozaki, M. (2009), "The Investigation on Using Unity3D Game Engine in Urban Design Study", ITB Journal of Information and Communication Technology, Vol. 3 No. 1, pp. 1-18.

Jönsson, J. (2017), Virtual Concept Development of Interior Lighting in the Automotive Industry, Linköping University.

Kosmadoudi, Z., Lim, T., Ritchie, J., Louchart, S., Liu, Y. and Sung, R. (2013), "Engineering design using game-enhanced CAD: The potential to augment the user experience with game elements", CAD Computer Aided Design, Vol. 45 No. 3, pp. 777-795.

Magica, R. (2014), Proteus : A Cruise Design Tool for the Future.

Schön, D. (1991), The Reflective Practitioner: How Professionals Think in Action, Ashgate Publishing Limited. Shiratuddin, M.F. and Thabet, W. (2002), "Virtual office walkthrough using a 3d game engine", International Journal of Design Computing, Vol. 4 No. 540, pp. 1329-7147.

Stappers, P. and Giaccardi, E. (2017), "Research through Design", Soegaard, M. and Friis-Dam, R. (Eds.), The Encyclopedia of Human-Computer Interaction, 2nd Edition., available at: http://www.interactiondesign.org/literature/book/the-encyclopedia-of-human-computer-interaction-2nd-ed/research-throughdesign.

Valencia-Romero, A. and Lugo, J.E. (2017), “An immersive virtual discrete choice experiment for elicitation of product aesthetics using Gestalt principles", Design Science, Vol. 3, p. e11.

Wördenweber, B., Boyce, P., Hoffman, D., Wallaschek, J., Boyce, P. and Hoffman, D. (2007), Automotive Lighting and Human Vision, Automotive Lighting and Human Vision, Springer-Verlag Berlin Heidelberg., available at: https://doi.org/10.1007/978-3-540-36697-3. 
